\title{
Heterogeneous Decomposition of a Hydrogen Sulfide-Methane Sour Mixture Flowing in a Rectangular Channel
}

\author{
J.C. Martínez ${ }^{1}$, F. Méndez*,2 and C. Treviño ${ }^{3}$ \\ ${ }^{1}$ Facultad de Ingeniería, Universidad Autónoma de Campeche 24030 Campeche, Mexico \\ ${ }^{2}$ Facultad de Ingeniería, Universidad Nacional Autónoma de México 04510 México DF, Mexico \\ ${ }^{3}$ Facultad de Ciencias, Universidad Nacional Autónoma de México 04510 México DF, Mexico
}

\begin{abstract}
In this work, we analyze the heterogeneous conversion process of hydrogen sulfide acid, $\mathrm{H}_{2} \mathrm{~S}$, to atomic hydrogen, $H^{0}$, in a binary gas mixture composed by the above acid component and methane gas. We consider that the sour mixture flows in a horizontal rectangular channel under a laminar, hydrodynamically fully-developed flow regime and the decomposition process takes place on the internal walls of the channel made by iron. The heterogeneous chemical activity on the metallic walls is conducted by a reactive scheme based on four reactions: one adsorption, two electrochemical, and an exothermic reaction needed to complete the conversion process. The longitudinal profiles of the species $\mathrm{H}_{2} \mathrm{~S}$ and $\mathrm{H}^{0}$ on the surfaces of the channel as functions of the nondimensional parameters involved in the present model, are shown. In addition, the surface coverages of the chemical products $H S^{-}, H^{+}$and $H^{0}$, together with the surface coverage of the vacancies derived from the chemical and electrochemical reactions are presented.
\end{abstract}

Keywords: Adsorption, Laminar flow, Electrochemistry, Hydrogen Sulfide, Heterogeneous reactions, Corrosion.

\section{INTRODUCTION}

The theoretical and experimental studies on hydrogen sulfide decomposition are a field of fundamental and practical importance because in numerous applications, this process can modify the operation and performance of different industrial devices. For instance, in the oil industry the socalled sour corrosion originated by the presence of hydrogen sulfide in oil-gas mixtures, can either accelerate or inhibit corrosion of iron tubes. The chemical and physical contact between sour gas mixtures and metallic surfaces yields that the corrosive component $\mathrm{H}_{2} \mathrm{~S}$ generates atomic hydrogen $H^{0}$ at the iron surface and this chemical specie penetrates diffusively into the metallic body, causing the cracking phenomenon with a complicated dynamic of fractures, indicating a clear evidence of the evolution of the corrosive process. In such cases, the hydrogen in the form of proton, $\mathrm{H}^{+}$, behaves as an ultra-fast diffuser and different effects like aqueous corrosion, electrolysis, electroplating, welding, etc compete to control the above diffusive process in many metals and alloys. Therefore, the simultaneous action of these effects modifies the mechanical properties of metals and alloys, reducing the average lifetime of metallic or dilute binary alloy structures. Glicksman [1] provides a basic discussion of the above aspects. On the other hand, abundant experimental evidence to show this corrosion type, including those cases for which the sour solution has important fractions of carbon dioxide, $\mathrm{CO}_{2}$, can be found in different ref-

*Address correspondence to this author at the Facultad de Ingeniería, Universidad Nacional Autónoma de México 04510 México DF, Mexico; Tel: +52-55-56228103; Fax: +52-55-56228106;

E-mail: fmendez@servidor.unam.mx erences. Chengqiang Ren et al. [2] clarified as the simultaneous influence of $\mathrm{H}_{2} \mathrm{~S}$ and $\mathrm{CO}_{2}$ can accelerate or inhibit the corrosion mechanism in circular steel tubes. Similar studies were reported by García et al. [3] and Houyi Ma et al. [4] to show the corrosive effects caused by the decomposition of $\mathrm{H}_{2} \mathrm{~S}$ on metallic pieces. Recently, Platonov et al. [5] have investigated the influence of some physico-chemical effects that can modify the dynamic gas extraction of hydrogen sulfide from aqueous solutions. These authors were focused on the control of the concentration of $\mathrm{H}_{2} \mathrm{~S}$, due to the fundamental interest to handle carefully petroleum products. Also in the petroleum industry, the use of metal oxides in reactors has various consequences. In these systems, the metal oxides can operate as non-catalyst and catalyst substrates for the processes of desulphurization and denitrogenation of oil-gas mixtures with high content of $\mathrm{H}_{2} \mathrm{~S}$. Wu et al. [6] studied experimentally the adsorption and desorption mechanisms of $\mathrm{H}_{2} \mathrm{~S}$ on a polycrystalline $\mathrm{UO}_{2}$ catalytic sample, operating under non-isothermal conditions. Their experimental results -obtained for different temperatures of the catalyst-, show the dissociative character of the component $\mathrm{H}_{2} \mathrm{~S}$. Similar results were reported by Reshetenko et al. [7], who investigated experimentally the heterogeneous reaction of decomposition of the hydrogen sulfide on bulk oxides $\gamma-\mathrm{Al}_{2} \mathrm{O}_{3}$, $\alpha-\mathrm{Fe}_{2} \mathrm{O}_{3}$ and $\mathrm{V}_{2} \mathrm{O}_{5}$ in the interval of temperature $500-900^{\circ} \mathrm{C}$. Generally, in these studies, the central objective is focused on the design of membrane reactors that offer evident advantages for the hydrogen production by means of the direct decomposition of $\mathrm{H}_{2} \mathrm{~S}$. Chan et al. [8] used a catalytic-membrane reactor containing a packed bed of 
$R u-M o$ sulfide catalyst to investigate the $H_{2} S$ decomposition. In order to validate their experimental results, they developed also a one-dimensional analytical model under nonisothermal conditions, examining in detail the effect of some hydrodynamic and transport properties on the decomposition of $\mathrm{H}_{2} \mathrm{~S}$ to $H^{0}$. They showed that Reynolds and Peclet numbers affect dramatically the conversion of $\mathrm{H}_{2} \mathrm{~S}$. A practical advantage of this type of catalytic reactors, among others, is that the recovery of $\mathrm{H}^{0}$ from $\mathrm{H}_{2} \mathrm{~S}$ represents a plausible economical alternative to the conventional Klaus process widely used in petroleum and minerals processing industries. A similar experimental analysis was reported by Startsev et al. [9] to show the decomposition of the $\mathrm{H}_{2} \mathrm{~S}$, under conditions of conjugate chemisorption and catalysis.

Recognizing that the study of the $\mathrm{H}_{2} \mathrm{~S}$ decomposition is very vast and complex, we have included some additional references [10-15] that offer fundamental details in other branches. However, the majority of the above works -except the work of Chan et al.-; give only experimental evidence of this decomposition in different applications. In an effort to provide a theoretical point of view, recently, Martinez et al. [16] studied the decomposition process for the hydrogen sulfide in a laminar stagnation flow. However, the longitudinal mass diffusion effects of the specie $H^{0}$ into the iron body, were not considering in the present work.

Therefore, in this analysis we treat theoretically the conversion process of hydrogen sulfide into atomic hydrogen for a binary gas mixture of methane and hydrogen sulfide, which is circulating under laminar conditions in a horizontal channel made of iron walls. Because the heterogeneous reactions of decomposition occurs at the internal surfaces of the channel, we anticipate that the mass transport of hydrogen sulfide, the heterogeneous conversion of $\mathrm{H}_{2} \mathrm{~S}$ to $\mathrm{H}^{0}$ on the surface of the metallic walls and the diffusive transport of this last specie through the walls represents a conjugate mass transfer problem, which defines the mixture-metallic surface interaction.

\section{THEORY}

\section{Mass Conservation Equations}

In Fig. (1) is illustrated the physical model, coordinate system and a sketch of the isothermal and laminar parallel flow. Because the gas mixture is found at room temperature $T_{\infty}$, the corrosion effects on iron walls are negligible, in a first approximation. We consider only a horizontal metallic wall, recognizing the symmetry condition of the problem. Here, $x$ and $y$ denote the horizontal and vertical axis, respectively and the origin is located just at the leading edge of the wall (with finite thickness $h$ and length $L$ ). Within this channel, a binary gas mixture of methane and hydrogen sulfide with mass concentrations $\bar{Y}_{\mathrm{CH}_{4}}$ and $\bar{Y}_{\mathrm{H}_{2} \mathrm{~S}}$, respectively; flows permanently with a well-known velocity profile $u$ and ambient temperature $T_{\infty}$. During the decomposition process, we also assume that the metallic walls are found in thermodynamics equilibrium with the gas mixture. The transport of hydrogen at the external surfaces of the walls follows a wellknown law of the type $F x^{n}$. For simplicity, we choose arbitrary values to these parameters. Due to heterogeneous electrochemical and chemical reactions on the iron wall (the detailed reaction mechanism will be given in the following subsection), the decomposition of $\mathrm{H}_{2} \mathrm{~S}$ to $\mathrm{H}^{0}$ is carried out continuously on the surface. In addition, we assume that in the region occupied by the binary mixture no exist homogeneous reactions of any type. Therefore, the developed velocity distribution and the mass conservation equation are the following:

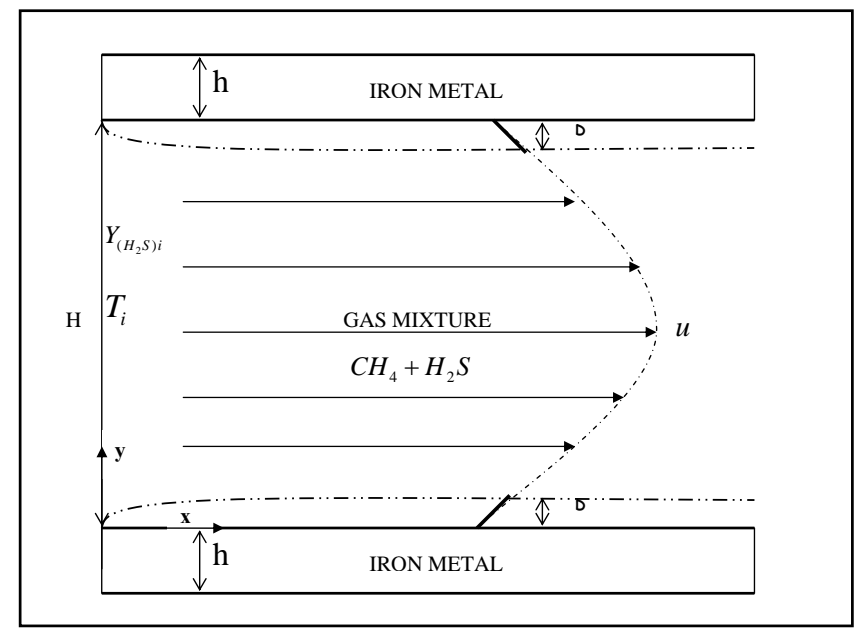

Fig. (1). Schematic diagram of the physical model.

$u=6 \bar{u}\left[\frac{y}{H}-\left(\frac{y}{H}\right)^{2}\right]$,

with

$\bar{u}=\frac{1}{12 \mu} \frac{d P}{d x} H^{2}$,

and

$u \frac{\partial \bar{Y}_{\mathrm{H}_{2} S}}{\partial x}=D_{\mathrm{H}_{2} S}\left(\frac{\partial^{2} \bar{Y}_{\mathrm{H}_{2} S}}{\partial x^{2}}+\frac{\partial^{2} \bar{Y}_{\mathrm{H}_{2} S}}{\partial y^{2}}\right)$.

In the above equations, $H$ is the width of the channel. $P$ and $\mu$ represent the pressure and dynamic viscosity of the mixture, respectively and obviously, we have considered a bi-dimensional laminar flow. During the decomposition process of $\mathrm{H}_{2} \mathrm{~S}$ to $\mathrm{H}^{0}$, we assume uniform values for the properties of the mixture. $D_{\mathrm{H}_{2} \mathrm{~S}}$ is the molecular diffusion coefficient of the specie $\mathrm{H}_{2} \mathrm{~S}$. Furthermore, we accept a global mass conservation between both species of the mixture, given by the relationship $\bar{Y}_{\mathrm{H}_{2} \mathrm{~S}}+\bar{Y}_{\mathrm{CH}_{4}}=1$. In order to solve the system of equations (1)-(3); we need appropriate boundary conditions. We suppose that, 


$$
x=0: \quad \bar{Y}_{H_{2} S}=\bar{Y}_{\left(H_{2} S\right) i} \quad \text { and } \quad y=\frac{H}{2}: \frac{\partial \bar{Y}_{H_{2} S}}{\partial y}=0
$$

where $\bar{Y}_{\left(\mathrm{H}_{2} \mathrm{~S}\right) \mathrm{i}}$ represents the molar concentration of $\mathrm{H}_{2} \mathrm{~S}$ at the entrance section of the channel. There are other boundary conditions related directly with the heterogeneous reactions at the internal surfaces of the metallic walls. At room temperature, the methane gas is inert and therefore cannot react with the iron surface. However, due to the presence of $\mathrm{H}_{2} \mathrm{~S}$ into the mixture, the contact of this specie with the metal can induce a spontaneous dissociative adsorption. Nelen et al. [13] and Lai et al. [17] have supported spectroscopic experimental evidence of this chemical dissociation, using metals in contact with a sour ambient composed basically by $\mathrm{H}_{2} \mathrm{~S}$ and operating at room temperature or lower than it.

The concept of dissociative adsorption has been widely studied in the past and specific details can be found in the work of Somorjai [18]. Therefore, we accept that the molecular dissociation that consumes slowly the hydrogen sulfide and inversely, creates atomic hydrogen at the surface of the iron. Hence, we propose the following mass transfer boundary conditions

$$
y=0: \quad \frac{\partial \bar{Y}_{H_{2} S_{w}}}{\partial y}-\frac{\omega_{9} \Gamma W_{G M}}{\rho_{G M} D_{H_{2} S}}=\frac{\partial \bar{Y}_{H_{w}^{0}}}{\partial y}-\frac{\omega_{11} \Gamma W_{I W}}{\rho_{I W} D_{H^{0}}}=0
$$

Here, $W_{i}$ is the molecular weight of specie $i$. The subscripts $G M$ and $I W$ denote gas mixture and iron wall, respectively. $\omega_{9}$ and $\omega_{11}$ are the surface reaction rates in units of mol of $\mathrm{H}_{2} \mathrm{~S}$ and $\mathrm{H}^{0}$ destructed and created by unit time and unit surface of the metallic wall, respectively. The parameter $\Gamma$ and appropriate laws for the reaction rates $\omega_{j}$ are defined in the following section and the subscript $j$ denotes the equation number corresponding to the heterogeneous reaction model defined by eqs. (8)-(11), given lines below. The above mass transportation boundary conditions represent the compatibility conditions to solve the conjugate mass transfer problem. Because the molar concentrations $\bar{Y}_{H_{2} S_{w}}$ and $\bar{Y}_{H_{w}^{0}}$ at the metallic wall are unknowns, we use a simple model for the molecular diffusion of hydrogen, taking into account the creation of this specie at the wall. Therefore, mass diffusion models that eventually could take into account the formation of unsaturable traps [1] are not considering in the present formulation. Under stationary conditions, we have that

$\frac{\partial^{2} \bar{Y}_{H_{w}^{0}}}{\partial x^{2}}+\frac{\partial^{2} \bar{Y}_{H_{w}^{0}}}{\partial y^{2}}=0$

together with,

$y=-h: \frac{\partial \bar{Y}_{H_{w}^{0}}}{\partial y}=F x^{n} ; x=0: Y_{H_{w}^{0}}=0$ and $\quad x=L: \frac{\partial Y_{H_{w}^{0}}}{\partial \chi}=0$

\section{Heterogeneous Reaction Model}

We adopt the same surface reaction model proposed by Martinez et al. [16], taking into account the simultaneous reactions for the dissociative adsorption of $\mathrm{H}_{2} \mathrm{~S}$ and the oxidation of the metallic surface. Nelen et al. [13] and Lai et al. [17] experimentally showed that this dissociative adsorption is selective, depending strongly on surface and temperature conditions. On the other hand, the first step to yield corrosion in metals comes from the anodic reaction of the metal with the sour environment. In this electrochemical reaction, there is an electronic interchange between the sour ambient and the iron metal, which easily conducts to the formation of $\mathrm{Fe}^{2+}$. Therefore, the reactive model is given by the following reactions,

$$
\begin{aligned}
& \mathrm{Fe} \rightarrow \mathrm{Fe}^{2+}+2 e^{-}, \\
& \mathrm{H}_{2} \mathrm{~S} \rightarrow \mathrm{HS}^{-}+H^{+}, \\
& \mathrm{Fe}^{2+}+\mathrm{HS}^{-} \rightarrow \mathrm{FeS}+\mathrm{H}^{+}, \\
& H^{+}+e^{-} \rightarrow H^{o} ;
\end{aligned}
$$

thus, electrochemically, when the iron wall is in contact with the sour mixture, the $\mathrm{HS}^{-}$ion is reduced to hydrogen atomic $H^{0}$, reacting with $F e^{2+}$ and in this form, ferrous sulfide, $\mathrm{FeS}$ is formed at the metal surface. However, it is very important to note that this simplified mechanism does not contain the adsorption-desorption of the product $\mathrm{FeS}$, as well as the adsorption of methane gas $\mathrm{CH}_{4}$, because these reactions require, in general, elevated temperatures. In addition, we assume a negligible generation rate for the $\mathrm{FeS}$ component. Furthermore, the specie $H^{-}$reacts only with $\mathrm{Fe}^{2+}$; otherwise, the generation of ions $\mathrm{H}^{+}$would be drastically reduced. On the other hand, the adsorption kinetic is given by a sticking probability, $S_{i}$ or accommodation coefficient, which represents the portion of the collisions with the surface that successfully leads to adsorption, [18]. Since we have only one adsorption reaction given by eq. (9), we denote this coefficient as $S_{i}=k_{9}$. On the other hand, the rate of collisions $Z_{w}$ can be computed by using the classical kinetic theory and the specie concentrations at the surface can be represented by the surface coverages $\theta_{i}$. It is defined as the ratio of the number of sites occupied by surface species $i$ to the total number of available sites. Considering the stationary case, the steady-state governing equations can be written as,

$\theta_{v}+\theta_{H S^{-}}+\theta_{H^{+}}+\theta_{H^{0}}=1$,

$\frac{d \theta_{H S^{-}}}{d t}=\omega_{9}-\omega_{10}=0$,

$\frac{d \theta_{H^{+}}}{d t}=\omega_{9}+\omega_{10}-\omega_{11}=0$ 
In addition, the mass diffusion of $\bar{Y}_{H_{w}^{0}}$ into the metallic wall modifies the surface coverage of this specie; then the corresponding reaction rate can be written as

$\frac{d \theta_{H^{0}}}{d t}=\omega_{11}-\frac{\rho_{I P} D_{H^{0}}}{\Gamma W_{I W}}\left|\frac{\partial \bar{Y}_{H_{w}^{0}}}{\partial y}\right|_{y=0}=0$.

In the above relationships, $\theta_{v}$ denotes the surface coverage of empty sites. In above equations, the reaction rates, $\omega_{j}$ are in $s^{-1}$ units and are defined by,

$\omega_{9}=k_{9} \theta_{v}^{2}, \omega_{10}=k_{10} \theta_{v} \theta_{H S^{-}}, \omega_{11}=k_{11} \theta_{H^{+}}$

and the kinetic factors $k_{j}$ as

$k_{9}=\frac{S_{0}^{2} P_{M}}{\Gamma\left(2 \pi W_{H_{2} S} R T\right)^{1 / 2}} \exp \left(-\frac{\Delta E_{9}}{R T}\right)$,

$k_{10}=A_{10} \exp \left(-\frac{\Delta E_{10}}{R T}\right)$,

$k_{11}=\frac{k_{B} T}{h_{P}} \exp \left(-\frac{n_{e 11} F \Delta V_{11}}{R T}\right)$.

In the above relationships, $S_{0}^{2}$ is the pre-exponential factor of the sticking coefficient between the hydrogen sulfide and the iron wall and is of order of 0.02 [19]. The superscript " 2 " on $S_{0}^{2}$ represents the dissociative character of this reaction. $\Gamma\left(=\frac{N_{V}}{N_{A}} \sim 3 x 10^{-27} \mathrm{~mol} / \mathrm{cm}^{2}\right)$ is the surface molar concentration and corresponds to the surface site density divided by the Avogadro's number. Here, $N_{V}=N_{a} \exp \left(\frac{-Q_{v}}{k_{B} T}\right)$ (in units of sites $/ \mathrm{cm}^{2}$ ) and is the total number of vacancies formed by thermal agitation per unit surface; $N_{a}$ is the total number of atoms in the solid, $Q_{v}$ is the energy required to form a vacancy that depends on the material and is of order of $\sim 1.08 \mathrm{eV}[20,21]$ and $N_{A}$ is the Avogadro's number $\left(=6.0225 \times 10^{23}\right.$ molecules $\left./ \mathrm{mol}\right) . R$ is the universal gas constant $(=8.3143 \mathrm{~J} / \mathrm{K} \mathrm{mol}) \cdot n_{e 11}=1$ and represents the number of participating electrons in the electrochemical reaction (11), $F$ is the constant of Faraday $\left(=9.6487 \times 10^{4} \mathrm{C} / \mathrm{mol}\right), \quad$ is the Boltzmann's constant $\left(=1.3805 \times 10^{-23} \mathrm{~J} / K\right)$ and $h_{P}$ is the constant of Planck (=6.6256 $\times 10^{-34} \mathrm{~J} s$ ). Furthermore, $T$ is the temperature in Kelvin and $P_{M}$ is the pressure of the mixture composed by $\mathrm{H}_{2} \mathrm{~S}$ and $\mathrm{CH}_{4}$. We consider that during the decomposition process, the mixture pressure remains unaltered. The concentrations, $\bar{Y}_{H_{2} S_{w}}, \bar{Y}_{H_{w}^{0}}$, etc. are the surface concentrations and are obtained after solving the gas-phase equations together with the governing equations for the surface coverage of the adsorbed species and the hydrogen diffusion into the metal.
The contact of the metallic wall with the sour mixture yields spontaneously a cell-electrochemical potential of magnitude $\Delta V_{8}$. Because at room conditions this electrochemical potential is of order of $0.44 \mathrm{~V}$ [22], with the aid of the Faraday's law, the corresponding electrochemical energy associated with this reaction is $\Delta E_{8}=n_{e 8} F \Delta V_{8}$ with $n_{e 8}=2$ (reciprocally, the electrochemical energy associated with reaction (11) is zero, because $\left.\Delta V_{11}=0\right)$. Therefore, we assume that this electrochemical energy is used to initiate the adsorption reaction, which is equivalent to write $\Delta E_{8}=n_{e 8} F \Delta V_{8} \sim \Delta E_{9}$. From equations (12)-(16) and after some simple algebraic manipulations can easily show that the surface coverage of the products are directly related to $\theta_{V}$ by means of the following relationships,

$$
\begin{aligned}
& \theta_{H S^{-}}=\frac{k_{9}}{k_{10}} \theta_{v}, \theta_{H^{+}}=\frac{2 k_{9}}{k_{11}} \theta_{v}^{2} \text { and } \\
& \theta_{H^{0}}=1-\theta_{v}-\frac{k_{9}}{k_{10}} \theta_{v}-\frac{2 k_{9}}{k_{11}} \theta_{v}^{2} .
\end{aligned}
$$

\section{NONDIMENSIONAL GOVERNING EQUATIONS}

The set of coupled governing equations (1)-(20) can considerably simplify by introducing some results of the boundary layer theory, [23] and appropriate dimensionless variables. In particular, we assume that near to the metallic wall there is a thin mass diffusion boundary layer of thickness $\delta_{D}$, caused by the weak consumption of $\mathrm{H}_{2} \mathrm{~S}$ at the wall. Because $\delta_{D} \ll L$ the velocity profile, eq. (1), within this zone can be linearized considering that $y \sim \delta_{D}$ and a simple dominant balance between the longitudinal mass convection and the transverse mass diffusion terms of eq. (3), yields that $\delta_{D} \sim L\left[(H / L)^{2} /\left(6 \operatorname{Re} S c_{H_{2} S}\right)\right]^{1 / 3} ;$ where $\operatorname{Re}=\bar{u} H / v$ and $S c_{\mathrm{H}_{2} \mathrm{~S}}=v / D_{\mathrm{H}_{2} \mathrm{~S}}$, and represent the Reynolds and Schmidt numbers, respectively; $v$ is the kinematic viscosity of the mixture. Under practical cases, it is common to assume that $\operatorname{Re} S c_{\mathrm{H}_{2} S} \gg>1$ and in this case, we recover that $\delta_{D} \ll L$ and therefore, the velocity profile, eq. (1) in this zone is of order of $\bar{u} y / H$, in a first approximation. On the other hand, the main advantage to use nondimensional variables is to reduce the number of involved physical parameters. Therefore, we define the following nondimensional variables

$$
\begin{aligned}
& \zeta=-\frac{y}{h}, \eta=\left(\left(\frac{H}{L}\right)^{2} \frac{1}{6 \operatorname{ReS} S c_{\mathrm{H}_{2} S}}\right)^{-1 / 3} \frac{y}{L}, \chi=\frac{x}{L} \\
& Y_{\mathrm{H}_{2} S}=\frac{\bar{Y}_{\mathrm{H}_{2} S}}{\bar{Y}_{\left(\mathrm{H}_{2} S\right) i}} \text { and } Y_{H^{0}}=\frac{\bar{Y}_{\mathrm{H}_{w}^{0}}}{\bar{Y}_{\left(\mathrm{H}_{2} S\right) i}} ;
\end{aligned}
$$

then, for the limit of $\operatorname{Re} S c_{\mathrm{H}_{2} S}>>1$, eq. (3) takes the form 
$\eta \frac{\partial Y_{H_{2} S}}{\partial \chi}=\frac{\partial^{2} Y_{H_{2} S}}{\partial \eta^{2}}$

where the longitudinal mass diffusion terms were omitted because they are of order of $\left(\operatorname{Re} S c_{\mathrm{H}_{2} S}\right)^{-2 / 3}$. The corresponding nondimensional boundary conditions are given by

$\chi=0, \forall \eta: Y_{H_{2} S}=1$,

$\eta \rightarrow \infty: Y_{H_{2} S}=1$,

and the compatibility conditions at the inner surface of the iron wall transform to

$$
\begin{aligned}
& \left.\frac{\bar{Y}_{\left(H_{2} S\right)_{i}}}{\delta_{D}} \frac{\partial Y_{H_{2} S}}{\partial \eta}\right|_{\eta=0}=\frac{k_{9} \Gamma W_{G M}}{\rho_{G M} D_{H_{2} S}} \theta_{v}^{2} \text { and } \\
& -\left.\frac{\bar{Y}_{\left(H_{2} S\right)_{i}}}{h} \frac{\partial Y_{H^{0}}}{\partial \zeta}\right|_{\zeta=0}=\frac{2 k_{9} \Gamma W_{I W}}{\rho_{I W} D_{H^{0}}} \theta_{v}^{2},
\end{aligned}
$$

that can be readily combined in only one relationship given by,

$$
\left.\frac{\partial Y_{H^{0}}}{\partial \zeta}\right|_{\zeta=0}=-\left.\frac{\varepsilon^{2}}{\alpha} \frac{\partial Y_{H_{2} S}}{\partial \eta}\right|_{\eta=0},
$$

where the nondimensional parameters $\alpha$ and $\varepsilon$ are defined as

$\alpha=\frac{\rho_{I W} W_{G M} h D_{H^{0}}}{2 \rho_{G M} W_{I W} L D_{H_{2} S}}\left(\left(\frac{H}{L}\right)^{2} \frac{1}{6 \operatorname{Re} S c_{H_{2} S}}\right)^{1 / 3}, \quad \varepsilon=\frac{h}{L}$

and $\alpha$ represents the competition between the rate of longitudinal molecular diffusion of hydrogen to the rate of hydrogen sulfide diffusion in the gas phase. This nondimensional parameter reflects the importance of the conjugate mass transfer between the mixture and the metallic wall.

On the other hand, the molecular diffusion of hydrogen into the iron wall, eq. (6), is described by the equation,

$\alpha \frac{\partial^{2} Y_{H^{0}}}{\partial \chi^{2}}+\frac{\alpha}{\varepsilon^{2}} \frac{\partial^{2} Y_{H^{0}}}{\partial \zeta^{2}}=0$,

while at $\zeta=1$ we have that,

$$
\left.\frac{\partial Y_{H^{0}}}{\partial \zeta}\right|_{\zeta=1}=-\beta \chi^{n} .
$$

where $\beta=F h L^{n} / \bar{Y}_{\left(H_{2} S\right)_{i}}$ is a nondimensional parameter that measures the intensity of the hydrogen gradient imposed at the external surface of the iron wall. In addition, the other boundaries of the iron wall are subject to,

$$
\chi=0: Y_{H^{0}}=0 \text { and } \chi=1: \frac{\partial Y_{H^{0}}}{\partial \chi}=0,
$$

ANALYTICAL SOLUTION FOR THE ASYMPTOTIC LIMIT $\alpha<1$ AND $\alpha \sim \varepsilon^{2}$

In this section, we obtain an analytical solution for the limit of $\alpha<<1$ with $\alpha \sim \varepsilon^{2}$. This case represents the majority of practical situations, because $\alpha \sim 1.25 \times 10^{-4}$. Due to the importance of this last nondimensional parameter and recognizing that $\alpha \sim \varepsilon^{2}$, we have called this limit as the thick diffusively wall regime. In addition, we assume that the aspect ratio $\varepsilon=h / L$ is smaller than unity, and therefore we neglect the first term of the left-hand side of eq. (28), in a first approximation. It means that the longitudinal mass diffusion effects at the metallic wall are confined to thin mass diffusion boundary layers at the edges of the wall. However, as the influence of these zones has only local influence are not considering here. Therefore, eq. (28) with the aid of the above approximation transforms to

$\frac{\partial^{2} Y_{H^{0}}}{\partial \zeta^{2}}=0$

and integrating once eq. (31), with the boundary conditions (26) and (29), we obtain that

$\frac{\partial Y_{H^{0}}}{\partial \zeta}=\left.\frac{\partial Y_{H^{0}}}{\partial \zeta}\right|_{\zeta=0}=-\left.\frac{\varepsilon^{2}}{\alpha} \frac{\partial Y_{H_{2} S}}{\partial \eta}\right|_{\eta=0}=\left.\frac{\partial Y_{H^{0}}}{\partial \zeta}\right|_{\zeta=1}=-\beta \chi^{n}$,

which express that the hydrogen gradient in the transverse direction is only a function of the longitudinal coordinate $\chi$.

Combining eq. (32) with the second relationship associated with the boundary conditions (25), we obtain an analytical solution for the surface coverage $\theta_{v}$ given by,

$\theta_{v}=\gamma \chi^{n / 2}$,

where $\gamma$ is a nondimensional parameter of order unity defined by $\gamma=\left(\rho_{I P} D_{H^{0}} F L^{n} / 2 k_{9} \Gamma W_{I P}\right)^{1 / 2}$. In our numerical estimations presented in the following section, $\gamma=0.0844$. Using the relationships (20), the other surface coverages can readily obtain,

$$
\begin{aligned}
& \theta_{H S^{-}}=\frac{k_{9}}{k_{10}} \gamma \chi^{n / 2}, \theta_{H^{+}}=\frac{2 k_{9}}{k_{11}} \gamma^{2} \chi^{n} \text { and } \\
& \theta_{H^{0}}=1-\left(1+\frac{k_{9}}{k_{10}}\right) \gamma \chi^{n / 2}-\frac{2 k_{9}}{k_{11}} \gamma^{2} \chi^{n} .
\end{aligned}
$$

On the other hand, integrating again eq. (32), we can obtain the distribution of the hydrogen into the metallic wall given by

$$
Y_{H^{0}}=\frac{\Gamma}{\Lambda} \theta_{H^{0}}(\chi)-\beta \chi^{n} \zeta
$$

In the above equation, the dimensionless concentration of the specie $i$ at the surface metallic wall is related with the

together with the compatibility condition (26). 
surface coverage through the relationship $Y_{i}=\frac{\Gamma}{\Lambda} \theta_{i}$. Here $\Lambda=l \rho_{I W} / W_{I W}$ and except by the other parameters defined in section $2, l$ is a characteristic length of the metallic microstructure [18].

In order to complete the set of analytical solutions associated with this decomposition process, the distribution of hydrogen sulfide at the surface of the wall -here denotes by $Y_{\left(\mathrm{H}_{2} \mathrm{~S}\right)_{W}}(\chi)^{-}$, is missing. We adopt the Lighthill's integral technique [23] to calculate this component at the iron surface. This technique is based on the superposition principle that can readily apply because eq. (22) is linear. In this case, the solution provides the nondimensional gradient of the hydrogen sulfide through the relationship,

$\left.\frac{\partial Y_{H_{2} S}}{\partial \eta}\right|_{\eta=0}=\chi^{-1 / 3}\left(Y_{\left(H_{2} S\right)_{W}}(\chi=0)+\int_{0}^{\chi}\left(1-\frac{\chi^{\prime}}{\chi}\right)^{-1 / 3} \frac{d Y_{\left(H_{2} S\right)_{W}}}{d \chi^{\prime}} d \chi^{\prime}\right)$

Finally and with the aid of equations (26), (29) and (32), we derive an integral equation to predict the distribution of the consumption of hydrogen sulfide at the surface of the metallic wall, given by,

$$
\beta \chi^{n}=\frac{\varepsilon^{2}}{\alpha} \chi^{-1 / 3}\left(Y_{\left(H_{2} S\right)_{W}}(\chi=0)+\int_{0}^{\chi}\left(1-\frac{\chi^{\prime}}{\chi}\right)^{-1 / 3} \frac{d Y_{\left(H_{2} S\right)_{W}}}{d \chi^{\prime}} d \chi^{\prime}\right)
$$

This integral equation is readily solving by means of the Abel's Integration and the resulting analytical solution takes the form [24]:

$$
Y_{\left(H_{2} S\right)_{W}}(\chi)=\frac{\sqrt{3} \alpha \beta}{2 \pi \varepsilon^{2}}\left(n+\frac{1}{3} \chi^{n-1 / 3}\right) .
$$

\section{RESULTS}

In this section, we present the figures that resulting from eqs. (33), (34), (35) and (38). All estimations were performed with the following data: Schmidt number $S c_{H_{2} S}=2.15, \quad \operatorname{Re}=3.0 \times 10^{3}$, room temperature of $T_{\infty}=300 \mathrm{~K}$ and the length of the channel $L=1 \mathrm{~m}$. In addition, $k_{9}=2.85 \times 10^{4} s^{-1}, k_{11}=6.25 \times 10^{12} s^{-1}$ and the sensibility factor $k_{9} / k_{10}=0.11$, [16]. Also, we assign arbitrary values to the parameters $n$ and $\alpha / \varepsilon^{2}$, taking into account that the first reflects the intensity of the extraction of hydrogen at the external surface of the iron wall of the channel and the second reveals the conjugate character of the mass diffusion. In Fig. (2) we have plotted the surface coverages of the product species $H^{-}, H^{+}, H^{0}$ and the vacancy surface coverage $\theta_{v}$ as functions of the nondimensional coordinate $\chi$, with $n=-1 / 6$ and $\alpha / \varepsilon^{2}=0.15$. The results show a direct dependency between the surface coverage of $H^{0}$ and the available vacancies on the surface. This clear competition, mainly, between both species controls the basic characteris- tics of the kinetic scheme. In contrast, the other species, $\mathrm{HS}^{-}$and $\mathrm{H}^{+}$have high reaction rates, particularly $\mathrm{H}^{+}$. In Fig. (3), we show the nondimensional concentration of hydrogen in the internal surface of the iron wall as a function of the nondimensional coordinate $\chi$, with $\alpha / \varepsilon^{2}=0.15$ and different values of the parameter $n$. In this figure is very important to note that for negative values of $n$, we obtain that the nondimensional concentration is increased, reflecting the most realistic situation because in this case, the corresponding nondimensional concentration of $\mathrm{H}_{2} \mathrm{~S}$ is decreasing. This last result may be appreciated from Fig. (4). In this figure, the surface concentration of hydrogen sulfide as function of the nondimensional coordinate $\chi$ and with $\alpha / \varepsilon^{2}=0.15$ and different values of the parameter $n$, is shown. In particular, for the value of $n=1 / 3$ is reached a limiting value that makes difficult the decomposition of $\mathrm{H}_{2} \mathrm{~S}$, because in this case the corresponding longitudinal concentration of the $H^{0}$ is decreasing. On the other hand, the case of $n=0$ requires a specific commentary. In this case and using eq. (29), the gradient of hydrogen at the external surface of the wall is uniform and the nondimensional concentration of $\mathrm{H}_{2} \mathrm{~S}$ represents a critical case because for positive values of $n$, the corresponding longitudinal profiles are saturated.

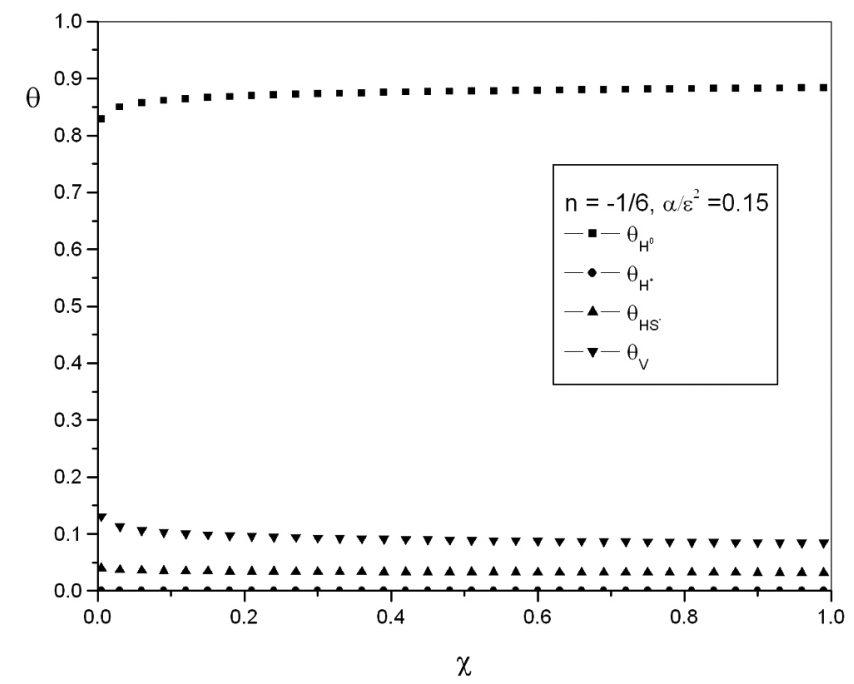

Fig. (2). Surface coverage of the products $H S^{-}, H^{+}, H^{0}$ and vacancies $\left(\theta_{H^{-}}, \theta_{H^{+}}, \theta_{H^{0}}\right.$ and $\left.\theta_{V}\right)$, as a function of the nondimensional coordinate $\chi$ and $n=-1 / 6, \alpha / \varepsilon^{2}=0.15$.

Fig. (5) shows the behavior of the hydrogen sulfide concentration as a function of the nondimensional coordinate $\chi$, $n=-1 / 6, \beta=0.5$ and arbitrary values for the nondimensional parameter $\alpha / \varepsilon^{2}$. The influence of the thick diffusively wall regime is clear: for decreasing values of the nondimensional parameter $\alpha / \varepsilon^{2}$, the corresponding surface concentration of $\mathrm{H}_{2} \mathrm{~S}$ diminishes, also. This result is evident because under this limit the transverse mass diffusion of $H^{0}$ is predominant. In Fig. (6), we show the transverse profiles 
of the nondimensional hydrogen concentration $Y_{H^{0}}$ at the iron wall as functions of the nondimensional transverse coordinate $\zeta$, for three different positions of the longitudinal coordinate $\chi$ and $n=-1 / 6, \beta=0.5, \alpha / \varepsilon^{2}=0.15$. In this same figure is evident that for increasing values of the longitudinal coordinate $\chi$, the slope of the curve is increased indicating a diffusion process weaker.

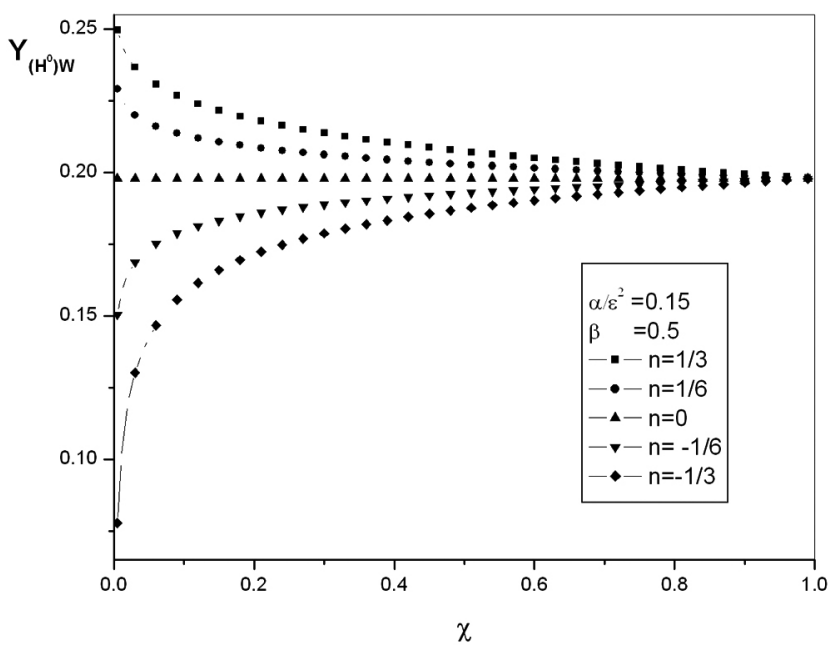

Fig. (3). Nondimensional hydrogen concentration at the internal surface of the metallic wall, $Y_{\left(H^{0}\right) W}$, as a function of the nondimensional coordinate $\chi$, different values of the parameter $n$ and $\beta=0.5, \alpha / \varepsilon^{2}=0.15$.

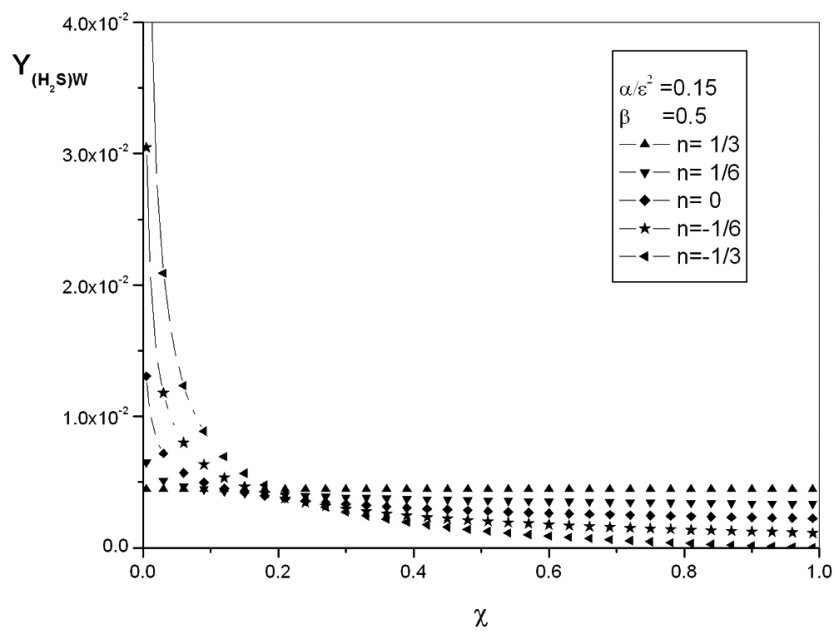

Fig. (4). Nondimensional hydrogen sulfide concentration at the internal surface of the metallic wall, $Y_{\left(H_{2} S\right) W}$, as a function of the nondimensional coordinate $\chi$, different values of the parameter $n$ and $\beta=0.5, \alpha / \varepsilon^{2}=0.15$.

Finally in Figs. (7 and 8), we show the influence of the nondimensional parameter $\beta$. In Fig. (7), the transverse profiles of the nondimensional hydrogen concentration $Y_{H^{0}}$ at the iron wall as functions of the nondimensional transverse coordinate $\zeta$, a only one position of the longitudinal coordinate $\chi=0.5$ and $n=-1 / 6, \beta=0.5, \alpha / \varepsilon^{2}=0.15$ and different values of $\beta$ are shown. In this figure, the growth of the hydrogen concentration along the metallic wall for decreasing values of the parameter $\beta$, indicates indirectly a better consumption of $\mathrm{H}_{2} \mathrm{~S}$ at the surface of the metallic wall. In fact, Fig. (8) verifies the above results.

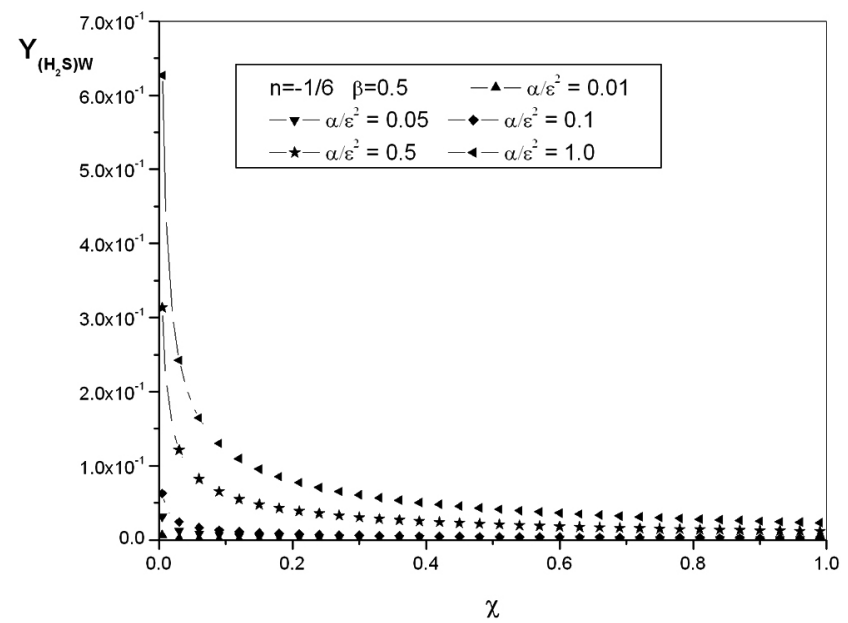

Fig. (5). Nondimensional hydrogen sulfide concentration at the internal surface of the metallic wall, $Y_{\left(\mathrm{H}_{2} \mathrm{~S}\right) \mathrm{W}}$, as a function of the nondimensional coordinate $\chi$, different values of the parameter $\alpha / \varepsilon^{2}$ and $\beta=0.5, n=-1 / 6$.

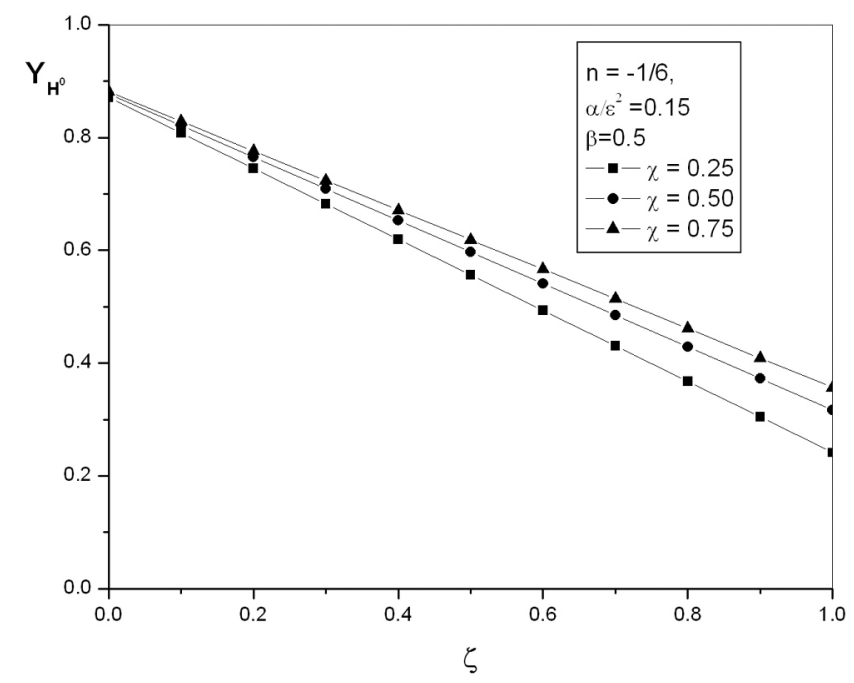

Fig. (6). Nondimensional hydrogen profile, $Y_{H^{0}}$, as a function of the nondimensional coordinate $\zeta$, different positions of the longitudinal coordinate $\chi$ and $n=-1 / 6, \alpha / \varepsilon^{2}=0.15, \beta=0.5$.

\section{CONCLUSION}

We have developed, in the present work, a simplified analytical model to understand the mechanism of heterogeneous decomposition of hydrogen sulfide to atomic hydrogen on the horizontal walls of a rectangular channel. The hydrogen sulfide is one of the components of a binary gas 


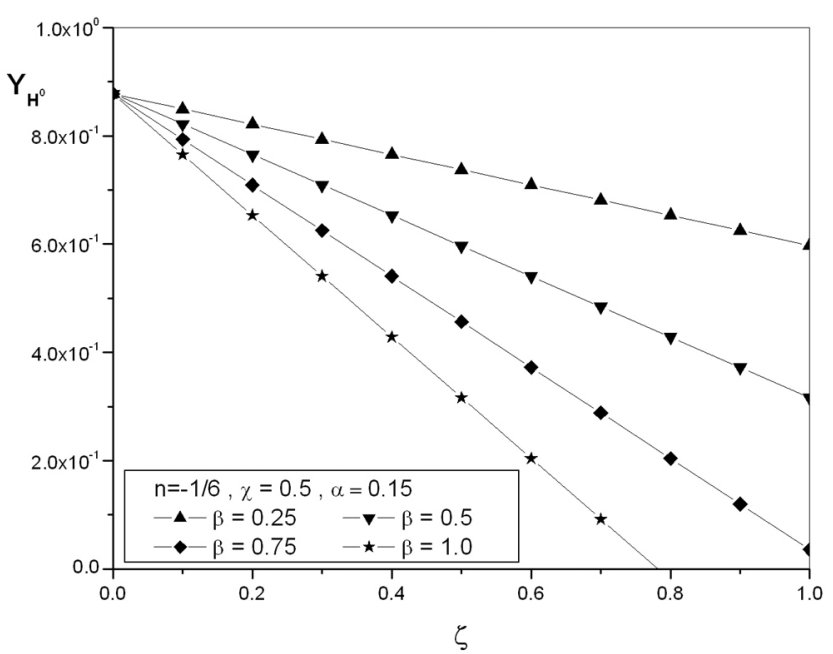

Fig. (7). Nondimensional hydrogen distribution, $Y_{H^{0}}$, as a function of the nondimensional coordinate $\zeta$, different values of the nondimensional parameter $\beta$ and $n=-1 / 6, \alpha / \varepsilon^{2}=0.15$ and a unique position of the nondimensional coordinate $\chi$.

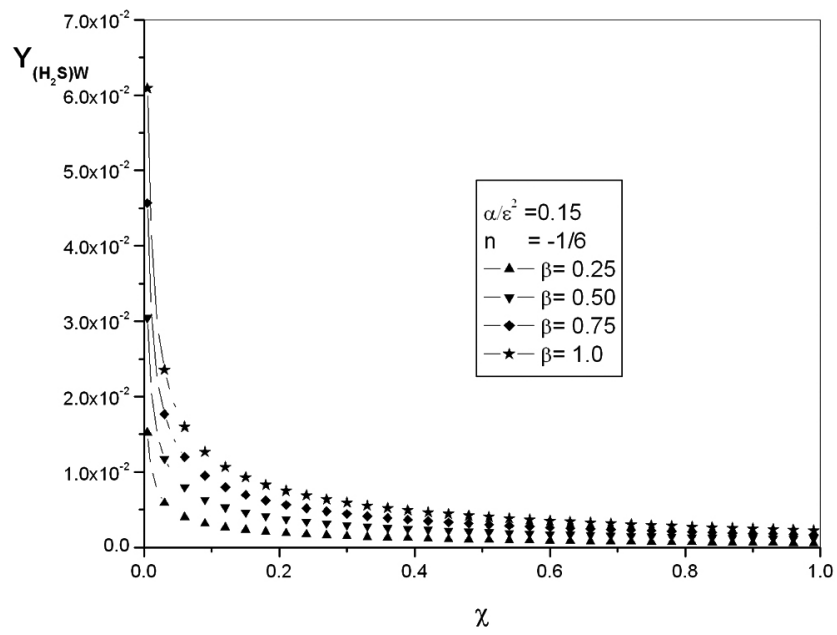

Fig. (8). Nondimensional hydrogen sulfide concentration at the internal surface of the metallic wall, $Y_{\left(\mathrm{H}_{2} \mathrm{~S}\right) W}$, as a function of the nondimensional coordinate $\chi, n=-1 / 6, \alpha / \varepsilon^{2}=0.15$ and different values of the nondimensional parameter $\beta$.

mixture; the other component is methane gas. This mixture is representative of sour-gas mixtures commonly found in the oil industry. For simplicity, we supposed that the binary mixture flows in a rectangular channel under a laminar and fully-developed flow regime and the metallic walls are made by iron. At room temperature, the metallic walls react slowly (the transient step that conducts to a steady-state regime was not considering in the present work) with this sour mixture, yielding $\mathrm{Fe}^{2+}$. In consequence, ion $\mathrm{Fe}^{2+}$ is recombined with $H^{-}$at the internal surfaces of the walls to conduct the process of generation of atomic hydrogen $H^{0}$. The specie $\mathrm{HS}^{-}$comes directly from the dissociative adsorption of $\mathrm{H}_{2} \mathrm{~S}$ and operates as a control to drive the reaction $\mathrm{Fe}^{2+}+\mathrm{HS}^{-} \rightarrow \mathrm{FeS}+\mathrm{H}^{+}$. Together with the proposed heterogeneous kinetic mechanism, the sticking surface coverage concept is used to predict -in the longitudinal direction of the wall-, how the available sites of the surface metal are occupied with the different products. Since a finite fraction of the generated hydrogen is conducted to the metal, we have included a simple process of diffusion of this specie. In this sense, the mathematical model is based on a conjugate mass transfer analysis, where the simultaneous interaction between the flow and the iron wall determines the longitudinal distributions of the heterogeneous surface reactions of the iron, dictated by the relationships (25).

Therefore, this simple analytical model predicts clearly the surface coverage of the involved products. However, we have not found experimental evidence in the specialized literature in order to support these theoretical predictions. Probably, this can be due to that the sour corrosion under laboratory conditions is forced to be carry out in short times increasing the temperature of the system. In addition, the theoretical predictions show that the electrochemical reactions (8) and (11) are, in general faster than other reactions (9) and (10), because the surface coverage of the transition specie $H^{+}$is smaller than the surface coverage of the other products. This result is appreciated in Fig. (1).

On the other hand, the theoretical predictions of the present work have been confirmed partially for some authors [3, $4,12,13,17]$. For instance, it is well known from these experimental studies that an elevated content of hydrogen sulfide in the mixture yields a drop of the surface coverage of $H^{0}$. Therefore, in order to explain the previous experimental results, we conclude that an equivalent figure to Fig. (8) for positive values of $n$ yields such condition, which was not included here.

Finally, it is important to note that the present model which is evaluated at room temperature only explains the heterogeneous decomposition of the specie $\mathrm{H}_{2} \mathrm{~S}$ and the corresponding generation and molecular diffusion of atomic hydrogen $\mathrm{H}^{0}$ into the metal. The trapping and cracking mechanisms that spread hydrogen in the form of protons, $\mathrm{H}^{+}$, through the surrounding lattice of the metal, accumulating internally $\mathrm{H}^{0}$ molecules in specific voids or traps to conduct the sour corrosion is outside of the main objectives of the present work.

\section{ACKNOWLEDGEMENTS}

This work has been supported by a research grant no. 43010Y of Consejo Nacional de Ciencia y Tecnología at Mexico.

\section{REFERENCES}

[1] M. E. Glicksman, Diffusion in Solids: Field Theory, Solid-State Principles and Applications. John Wiley and Sons, New York, 2000.

[2] C. Ren, D. Liu, Z. Bai, and T. Li, "Corrosion behavior of oil tube steel in simulant solution with hydrogen sulfide and carbon dioxide", Mater. Chem. Phys., vol. 93, pp. 305-309, October 2005.

[3] L. A. C. J. García, C. J. B. M. Joia, E. M. Cardoso, and O. R. Mattos, "Electrochemical methods in corrosion on petroleum industry: laboratory and field results", Electrochim. Acta, vol. 46, pp. 38793886, August 2001. 
[4] H. Ma, X. Cheng, G. Li, S. Chen, Z. Quan, S. Zhao, and L. Niu. "The influence of hydrogen sulfide on corrosion of iron under different conditions", Corros. Sci., vol. 42, pp. 1669-1683, October 2000.

[5] I. A. Platonov, D. R. Ismagilov, S. Yu. Kudryaashov I.N. Smygina, L.A. Onuchak, and V.G. Berezkin "Generating gas flows with a constant trace concentration of hydrogen sulfide", J. Anal. Chem., vol. 61, pp. 52-57, January 2006.

[6] Q. Wu, B. V. Yakshinskiy, T. Gouder, and T. E. Madey, " $\mathrm{H}_{2} \mathrm{~S}$ adsorption on polycrystalline $\mathrm{UO}_{2}{ }^{\prime \prime}$, Catal. Today, vol. 85, 291301, October 2003.

[7] T. V. Reshetenko, S. R. Khairulin, Z. R. Ismagilov, and V. V. Kuznetsov, "Study of the reaction of high-temperature $\mathrm{H}_{2} \mathrm{~S}$ decomposition on metal oxides $\left(\gamma-\mathrm{Al}_{2} \mathrm{O}_{3}, \mathrm{\alpha}-\mathrm{Fe}_{2} \mathrm{O}_{3}, \mathrm{~V}_{2} \mathrm{O}_{5}\right)$ ", Int. J. Hydrogen Energy, vol. 27, pp. 387-394, April 2002.

[8] P. P. Y. Chan, K. Vanidjee, A. A. Adesina, and P. L. Rogers, "Modeling and simulation of non-isothermal catalytic packed bed membrane reactor for $\mathrm{H}_{2} \mathrm{~S}$ decomposition", Catal. Today, vol. 63, pp. 379-385, December 2000.

[9] A. N. Startsev, I. I. Zakharov, O. V. Voroshina, A. V. Pashigreva, and V. N. Parmon, "Low-temperature decomposition of hydrogen sulfide under the conditions of conjugate chemisorption and catalysis", Dokl. Phys. Chem., vol. 399, pp. 283-286, November 2004.

[10] S. G. Addepalli, N. P. Magtoto, and J. A.Kelber, " $\mathrm{H}_{2} \mathrm{~S}$ adsorption and the effect of sulfur on the oxidation of $\mathrm{Ni}_{3} \mathrm{Al}$ (111)", Surf. Sci., vol. 458, pp. 123-134, June 2000.

[11] B. G. Ateya, F. M. Al-Kharafi, R. M Abdallah, and A. S. Al-Azab, "Electrochemical removal of hydrogen sulfide from polluted brines using porous flow through electrodes", J. Appl. Electrochem., vol. 35, pp. 297-303, March 2005.

[12] M. Çakmak, and G. P. Srivastava, "Ab-initio study of the adsorption of $\mathrm{H}_{2} \mathrm{~S}$ onto the $\mathrm{Si}$ (001) surface", Surf. Sci., vols. 433-435, pp. 420-424, August 1999.

[13] L. M. Nelen, K. Fuller, and C. M. Greenlief, "Adsorption and decomposition of $\mathrm{H}_{2} \mathrm{~S}$ on the $\mathrm{Ge}(100)$ surface", Appl. Surf. Sci., vol. 150, pp. 65-72, August 1999.
[14] H. Ohashi, H. Ohya, M. Aihara, Y. Negishi, and S. I.Semenova, "Hydrogen production from hydrogen sulfide using membrane reactor integrated with porous membrane having thermal and corrosion resistance", J. Membr. Sci. , vol. 146, pp. 39-52, July 1998.

[15] C. Thaeron, D. J. Parrillo, S. Sicar, P. F. Clarke, M. Paranjape, and B. B. Pruden, "Separation of hydrogen sulfide-methane mixtures by selective surface flow membrane", Sep. Purif. Technol., vol. 15, pp. 121-129, March 1999.

[16] J. C. Martínez, , F. Méndez, and C. Treviño, "Theoretical analysis for the heterogeneous decomposition of hydrogen sulfide to hydrogen on an iron-metallic plate in a laminar stagnation-point flow", Appl. Surf. Sci., vol. 253, pp. 2327-2335, December 2006.

[17] Y.-H. Lai, Ch.-T. Yeh, Y.-H. Lin, and W.-H. Hung, "Adsorption and thermal decomposition of $\mathrm{H}_{2} \mathrm{~S}$ on $\mathrm{Si}$ (100)", Surf. Sci., vol. 519, pp. 150-156, November 2002.

[18] G. A. Somorjai, Introduction to Surface Chemistry and Catalysis. John Wiley \& Sons, New York, 1994.

[19] B. Deng, S. Hu, T. M. Whitworth, R. Lee, "Trichloroethylene Reduction on Zero Valent Iron: Probing Reactive versus Nonreactives Sites" in Chlorinated Solvent and DNAPL Remediation. Innovative Strategies for Subsurface Cleanup, S. M. Henry and S. D. Warner Eds. ACS Symposium Series 837 USA: Oxford University Press, 2003, pp. 181-205.

[20] W.D. Callister Jr., Materials Science and Engineering: An Introduction. $5^{\text {th }}$ edition, Wiley and Sons, New York, 1999.

[21] V. V. Ogorodnikov, A. N. Rakitskii, and Yu. I. Rogovoi, "Calculation of the vacancy formation energy of metals" Powder Metall. Met. Ceram., vol. 27, pp. 55-60, January 1988.

[22] J. O'M. Bockris, and A. K. N. Reddy, Modern Electrochemistry. vol. 2, pp. 1282, Plenum Press, New York, 1970

[23] H. Schlichting, Boundary Layer Theory. McGraw-Hill, $6^{\text {th }}$ edition, New York, 1968.

[24] G. Arfken, Mathematical Methods for Physicists. Third Edition, Academic Press, Inc., New York, 1985. 